TRABAJO EN PROGRESO

\title{
Análisis de las interacciones sociales y humano- computadora durante el diseño colaborativo de videos interactivos
}

\author{
Juan Antonio Martínez Herrera, Maria Susana Avila \\ Garcia, Marco Bianchetti
}

Publicado: 21 Septiembre 2016

\begin{abstract}
Resumen
El presente trabajo muestra un análisis cualitativo de las interacciones sociales y humano-computadora de un grupo de estudiantes que han presentado conflictos sociales al momento de resolver actividades en grupo relacionadas con la creación de videos interactivos. Este grupo se caracteriza por la diferencia de edad de sus miembros y el nivel de conocimiento en cuanto al manejo de tecnologías de información. Las situaciones de conflicto comúnmente suelen presentarse cuando los miembros del equipo, con poco nivel de conocimiento en el área de tecnologías de información, trabajan con aquellos que poseen un mayor nivel. Dado que los estudiantes trabajan en sus computadoras portátiles, existe un sentido de pertenencia del dispositivo y del área de trabajo al momento de realizar las tareas. El objetivo de este trabajo es el de analizar los cambios en las interacciones sociales y humano-computadora de los estudiantes al momento en que resuelven actividades colaborativas en una pantalla multitáctil en relación con una computadora portátil. Este trabajo se enfoca en aspectos de sentido de pertenencia del espacio de trabajo, la interacción con cada uno de los elementos de la aplicación, así como en el intercambio de ideas y conocimiento.
\end{abstract}

Palabras clave: Interacción Humano-Computadora; Pantalla Multitáctil; Interacción Social; Videos Interactivos; Enseñanza del Inglés.

\section{Introducción}

Actualmente en el departamento de estudios multidisciplinarios (DEM) sede Yuriria de la Universidad de Guanajuato se ofrece la carrera de Licenciatura en la Enseñanza del Inglés (LEI), en la que los estudiantes obtienen competencias en cuanto al uso de tecnologías de información para dar soporte la enseñanza del inglés. Los grupos de estudiantes pertenecientes a esta carrera son muy diversos destacando dos aspectos: edad y su capacidad para

Martínez Herrera, JA., Avila Garcia, MS., Bianchetti, M.

Universidad de Guanajuato

DICIS

Av. Universidad S/N

Col. Yacatitas, Yuriria Gto.

ja.martinezherrera@ugto.mx, susana.avila@ugto.mx,mb@ugto.mx el manejo de tecnologías de información. Las edades de los estudiantes oscilan entre los 18 y 50 años de edad lo cual provoca que las personas mayores puedan llegar a sentirse intimidadas por los jóvenes, principalmente al momento hacer uso de herramientas tecnológicas. Por otra parte, los conocimientos de los estudiantes de estos grupos en cuanto a tecnología es muy variado, en algunos casos dominan completamente la tecnología contrastando con aquellos que desconocen completamente el manejo de esta lo cual ha llegado a provocar roces entre los estudiantes debido a esta situación. Una vez observada esta situación se decidió analizar el impacto de una mesa multitáctil en el desarrollo de actividades colaborativas, y analizar las interacciones sociales y humano computadora de un grupo de participantes pertenecientes a uno de los grupos anteriormente mencionados. El trabajo se enfoca en analizar las diferencias mientras resuelven una actividad utilizando una computara portátil y una mesa multitáctil. En esta mesa pueden interactuar más de dos personas al mismo tiempo, debido a su capacidad multitáctil los usuarios pueden acceden a elementos que son mostrados en pantalla desde diferentes puntos. Estas pantallas pueden ser colocadas de manera vertical, horizontal, y hasta en el piso. El diseño de aplicaciones para pantallas multitáctiles tiene que tener en consideración varios factores entre los que se encuentra la posición de los usuarios de tal manera que puedan acceder a los elementos de la pantalla. Existen trabajos donde se estudia la interacción social con pantallas multitáctiles, por ejemplo, se han reportado casos donde grupos de extraños socializan y se divierten entre ellos, incluso cuando iniciaron su interacción en diferente momento [1]. Estos tipos de interacciones han sido estudiadas en grupos de niños, por ejemplo, el prototipo SEESAW [2], fue diseñado para mejorar la interacción entre niños al momento de realizar actividades en grupo. El presente trabajo, se centra en estudiar el impacto del uso de una pantalla multitáctil en las interacciones entre individuos de diferente rango de edad y diferentes conocimientos de herramientas de tecnologías de información que han presentado ciertos conflictos en el pasado. La pantalla se utiliza de manera horizontal para promover el trabajo colaborativo, como se sugiere en [3], al momento en que desarrollan actividades de diseño de videos interactivos. 


\section{Metodología}

\subsection{Participantes}

9 estudiantes en un rango de edad entre 20 y 25 años, 2 en un rango de entre 25 a 30 años, una persona mayor de 40 años. Se obtuvo el consentimiento previo de los participantes para ser videograbados y utilizar los resultados de las actividades para este trabajo.

\subsection{Material}

Cámara de video, pantalla multitáctil 3M-C4667PW, Computadora personal, plataforma EDPUZZLE para desarrollo de videos interactivos en línea

\subsection{Procedimiento}

Fueron definas dos sesiones de grabación, en la primera los participantes resolvieron una actividad la cual consistía en diseñar una videotribia en la plataforma Edpuzzle utilizando una computadora personal en grupos de tres, en la segunda los estudiantes resolvieron una actividad en la misma plataforma usando una pantalla multitáctil, debido a que la plataforma no estaba diseñada para un ambiente multitáctil esta funcionalidad no fue explotada al máximo. Las sesiones fueron grabadas en video, el cual fue analizado y editado para ocultar la identidad de los participantes.

\section{Diseño experimental}

La primera sesión se realizó en un aula donde los participantes formaron grupos, cada grupo fue ubicado en una parte del aula, se instaló una videocámara en la parte frontal de tal forma que pudiera captar a todos los participantes, en esta sesión los participantes resolvieron una actividad en una plataforma en línea llamada EDPUZZLE. Esta herramienta permite la creación de videotibias las cuales son resueltas por otros estudiantes. Durante el desarrollo de la actividad los participantes utilizaron una computadora portátil, no existieron restricciones para los participantes, ni se les indico quien debía hacer uso de la computadora ni en qué momento. Se capturó en video el desarrollo de toda la actividad (ver Figura 1).

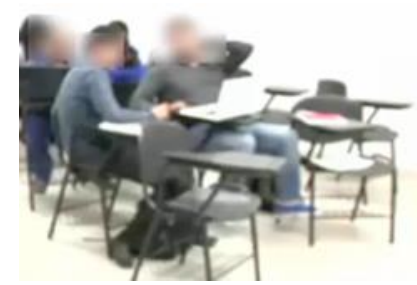

Figura 1. Video Sesión 1

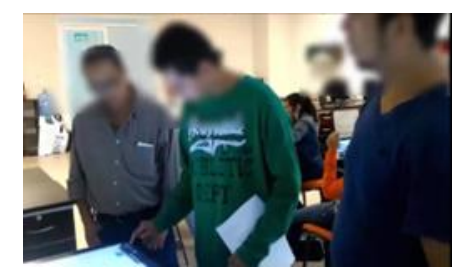

Figura 2. Video Sesión 2

En la segunda sesión los participantes realizaron una actividad en una pantalla multitáctil utilizando la misma plataforma se utilizó un espacio más abierto que permitiera la movilidad de los participantes, se tomaron turnos debido a que solamente se contaba con una pantalla multitáctil. Un participante fungió como camarógrafo utilizando un teléfono celular, esto con la finalidad de que no se sintieran invadidos y actuaran de la manera más natural posible. El video obtenido en ambas sesiones fue almacenado dentro de la institución y editado para asegurar la identidad de los participantes. En ambas sesiones se contó con un moderador que intervenía en caso de que los participantes se estancaran con algún elemento de la actividad (ver Figura 2).

\section{Análisis de los datos}

Una vez obtenidas las sesiones de grabación, el video obtenido fue observado varias ocasiones para detectar patrones de comportamiento y definir códigos de interacción, estos códigos representan una interacción o una acción de los usuarios entre ellos mismos o con el dispositivo que están utilizando; estos códigos fueron registrados en rúbricas en base a los códigos reportados en [4]. Una vez establecidos estos códigos se realizó un análisis exhaustivo del video para registrar la ocurrencia de cada interacción en las rúbricas para de esta manera cuantificar su ocurrencia. Los códigos definidos para registrar las interacciones entre participantes y dispositivos son:

- Participante-Dispositivo: Uno o más participantes interactúan con el dispositivo que se encuentran utilizando.

- Participante-Participante: Los participantes dialogan entre ellos, expresan ideas o comentarios sobre la actividad, o solicita ayuda a otro participante.

- Participante-Nada: Los participantes realizan acciones no relacionadas con la actividad.

- Participante-Maestro: Los participantes solicitan ayuda al moderador de la actividad

- Maestro-Participante: El moderador interviene en caso que los participantes se encuentren estacados en algún punto de la actividad.

\section{Resultados preliminares}

El presente trabajo se encuentra en desarrollo, y los resultados preliminares muestran que existe una mayor interacción con la pantalla multitáctil comparado con la computadora portátil proporcionando la posibilidad de crear videos interactivos con una participación más equitativa y menos limitaciones en cuanto al espacio físico de trabajo que estos dispositivos ofrecen. Se observa también que las interacciones sociales cambian entre los individuos y se pudo notar un alto sentido de pertenencia de espacio sobre la pantalla en ciertos participantes lo cual pudo haber sido resultado del dominio de conocimiento sobre cómo realizar la actividad por los participantes. Actualmente se están analizando las interacciones de los individuos con los elementos de la aplicación de tal manera que se pueda entender que elementos son los más utilizados y con los resultados obtenidos informar sobre una serie de recomendaciones para el diseño de aplicaciones para la creación y edición de videos interactivos en mesas multitáctiles basadas en consideraciones de distribución del espacio físico, colocación de elementos de aplicación como menús, etc.

\section{Agradecimientos}

Juan Antonio Martínez Herrera agradece al Consejo Nacional de Ciencia y Tecnología (CONACyT) por el apoyo brindado con la beca no. 427538 .

\section{Referencias}

[1] Peltonen, P., et al., It's Mine, Don't Touch!: interactions at a large multi-touch display in a city centre, in Proceedings of 
the SIGCHI Conference on Human Factors in Computing Systems. 2008, ACM: Florence, Italy. p. 1285-1294.

[2] Yang, C. and S.J. Wang, Seesaw: An Interactive Display to Facilitate Social Interaction. Procedia Technology, 2015. 20: p. 104-110

[3] Rogers, Y.a.L., S., Collaborating Around Large Interactive Displays: Which Way is Best to Meet?, in Interacting with Computers. 2004
[4] Mercier, E., G. Vourloumi, and S. Higgins, Student interactions and the development of ideas in multi-touch and paper-based collaborative mathematical problem solving. British Journal of Educational Technology, 2015: p. n/a-n. 